\title{
Interleukin 1-Beta (IL-1 $\beta$ ) Production by Innate Cells Following TLR Stimulation Correlates With TB Recurrence in ART-Treated HIV-Infected Patients
}

\author{
Christina Thobakgale, PhD, ${ }^{*}+\neq$ Kewreshini Naidoo, MSc, * Lyle R. McKinnon, PhD, $\$ \| \dagger$ \\ Lise Werner, MSc, $†$ Natasha Samsunder, MSc, $†$ Salim Abdool Karim, MD, $†$

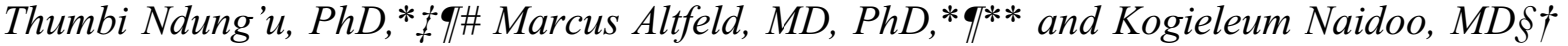

\begin{abstract}
Background: Tuberculosis (TB) remains a major cause of global morbidity and mortality, especially in the context of HIV coinfection because immunity is not completely restored following antiretroviral therapy (ART). The identification of immune correlates of risk for TB disease could help in the design of hostdirected therapies and clinical management. This study aimed to identify innate immune correlates of TB recurrence in HIV+ ART-treated individuals with a history of previous successful TB treatment.
\end{abstract}

Methods: Twelve participants with a recurrent episode of TB (cases) were matched for age, sex, time on ART, pre-ART CD4 count with 12 participants who did not develop recurrent TB in 60 months of follow-up (controls). Cryopreserved peripheral blood mononuclear cells from time-points before TB recurrence were stimulated with ligands for Toll-like receptors (TLR) including TLR-2, TLR-4, and TLR-7/8. Multicolor flow cytometry and intracellular cytokine staining were used to detect IL- $1 \beta$, TNF- $\alpha$, IL-12, and IP10 responses from monocytes and myeloid dendritic cells (mDCs).
Results: Elevated production of IL-1 $\beta$ from monocytes following TLR-2, TLR-4, and TLR-7/8 stimulation was associated with reduced odds of TB recurrence. In contrast, production of IL-1 $\beta$ from both monocytes and mDCs following Bacillus CalmetteGuérin (BCG) stimulation was associated with increased odds of TB recurrence (risk of recurrence increased by $30 \%$ in monocytes and $42 \%$ in mDCs, respectively).

Conclusion: Production of IL-1 $\beta$ by innate immune cells following TLR and BCG stimulations correlated with differential TB recurrence outcomes in ART-treated patients and highlights differences in host response to TB.

Key Words: Toll-like receptor, monocytes, myeloid dendritic cells, HIV/TB coinfection, TB recurrence

(J Acquir Immune Defic Syndr 2017;74:213-220)

\section{INTRODUCTION}

Tuberculosis (TB) is a global health problem causing nearly 10 million new infections every year. In particular, TB

Received for publication July 24, 2015; accepted August 31, 2016.

From the *HIV Pathogenesis Programme, Doris Duke Medical Research Institute, University of KwaZulu-Natal, Durban, South Africa; $†$ Centre for the AIDS Programme of Research in South Africa, Nelson R Mandela School of Medicine, University of KwaZulu-Natal, Durban, South Africa; †Ragon Institute of Massachusetts General Hospital, Massachusetts Institute of Technology and Harvard University, Boston, MA; §MRC HIV-TB Pathogenesis and Treatment

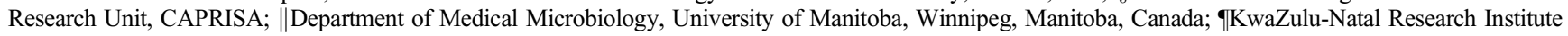
for Tuberculosis and HIV, Nelson R Mandela School of Medicine, University of KwaZulu-Natal, Durban, South Africa; \#Max Planck Institute for Infection Biology, Berlin, Germany; and **Heinrich-Pette-Institute, Leibniz Institute for Experimental Virology, Hamburg, Germany.

Supported by Doris Duke Charitable Foundation and the South African Medical Research Council. The TRUTH study was supported by the Howard Hughes Medical Institute, Grant \# 55007065 and the Centers for Disease Control and Prevention (CDC) Cooperative Agreement Number UY2G/PS001350-02. The research infrastructures to conduct this trial, including the data management, laboratory and pharmacy cores, were established through the US National Institutes for Health's Comprehensive International Program of Research on AIDS grant (CIPRA, Grant \# AI51794). Kogieleum Naidoo was supported by the Columbia University-South Africa Fogarty AIDS International Training and Research Program (AITRP, Grant \# D43 TW000231). Patient care was supported by the KwaZulu-Natal Department of Health and the US President's Emergency Plan for AIDS Relief (PEPFAR).

T.N. is an International Early Career Scientist of the Howard Hughes Medical Institute (Grant \# 55007427) and received additional funding from the South African Research Chairs Initiative and the Victor Daitz Foundation (Grant \# 64809). The remaining authors have no conflicts of interest to disclose.

Supplemental digital content is available for this article. Direct URL citations appear in the printed text and are provided in the HTML and PDF versions of this article on the journal's Web site (www.jaids.com).

Its contents are solely the responsibility of the authors and do not necessarily represent the official views of either the Howard Hughes Medical Institute or the Centers for Disease Control and Prevention (CDC). The research infrastructures to conduct this trial, including the data management, laboratory and pharmacy cores, were established through the US National Institutes for Health's Comprehensive International Program of Research on AIDS grant (CIPRA, Grant \# AI51794).

The funding sources listed here did not have any role in the analysis or preparation of the data in this manuscript, nor was any payment received by these or other funding sources for this manuscript.

Correspondence to: Christina Thobakgale, PhD, HIV Pathogenesis Programme, Doris Duke Medical Research Institute, Nelson R Mandela School of Medicine, University of KwaZulu-Natal, Room 114, Durban 4001, South Africa (e-mail: thobakgalec@ukzn.ac.za).

Copyright (C) 2016 The Author(s). Published by Wolters Kluwer Health, Inc. This is an open-access article distributed under the terms of the Creative Commons Attribution-Non Commercial License 4.0 (CCBY-NC), where it is permissible to download, share, remix, transform, and buildup the work provided it is properly cited. The work cannot be used commercially without permission from the journal. 
is the most common cause of death in HIV-infected individuals in Africa. ${ }^{1}$ Although TB infects approximately one-third of the global population, the vast majority of these individuals readily contain TB infection, with only $5 \%-10 \%$ developing active TB during their lifetime. A substantially higher risk of active TB does however exist in immunosuppressed individuals especially those with HIV coinfection. ${ }^{2}$

South Africa has the highest burden of HIV and TB coinfection globally. Studies show that TB recurrence rates greatly depend on TB incidence and HIV prevalence. ${ }^{3-5}$ A study conducted in South Africa reported that recurrent TB after successful treatment was up to 4 times that of new TB disease, indicating a high risk of TB recurrence in people who experienced a TB episode. ${ }^{5}$ Thus, factors that predispose individuals to TB acquisition may continue to play a role for susceptibility to TB recurrence as well.

Millennia of coevolution with the host have equipped TB with many ways to elude natural immune defenses and transit into a stage of relative dormancy. ${ }^{6-9}$ Satisfactory control of TB would be best achieved using effective preventative TB vaccines. Progress in new TB vaccine development has been hampered by incomplete understanding of correlates of natural protection against $\mathrm{TB}$ that successful vaccines should emulate.

The continuum of host-pathogen interaction following infection with Mycobacterium tuberculosis (Mtb) to $\mathrm{Mtb}$ disease traverses innate immune, adaptive immune, quiescent, and active replicating phases of infection. This continuum may extend beyond successful Mtb treatment when the cycle of TB reinfection may occur. In approximately $5 \%$ of treated patients, live Mtb infection persists, which may subsequently cause a relapse of TB disease; whereas in others, reinfection with Mtb may cause subsequent TB disease. ${ }^{10}$ Several biomarkers have been described that reflects the biology of TB infection and or disease; however, to date no biomarker that accurately predicts latent, active TB or recurrent TB exists.

Although the innate immune response is the first to encounter Mtb upon exposure, this arm of the immune system has not been well studied in the setting of TB in humans. Key immune cells that are the first contact include macrophages and dendritic cells, and these cells express Toll-like receptors (TLRs) that recognize specific signatures on pathogens, initiating signaling pathways that trigger production of innate immune effector molecules, cytokines, and chemokines. This response not only dictates the activity of the innate immune system but is also critical in initiating the adaptive responses to Mtb likely leading to successful long-term containment. ${ }^{11}$ Previous studies have implicated TLR-2 and TLR-4 in the direct recognition of Mtb. ${ }^{12,13}$ Data from a case-population study found a significant association between polymorphisms in a negative regulator gene of TLR/IL-1R signaling with increased TB susceptibility. ${ }^{14}$ Additionally, an additive risk of TB susceptibility was observed with coinheritance of these polymorphisms and previously identified TLR-risk alleles.

Although antiretroviral therapy (ART) restores CD4 T-cell numbers, effects of HIV infection on TB immunity are only partially reversed. ${ }^{15}$ For example, reservoirs of HIV harbored in tissue macrophages make it difficult for eradication by ART and may lead to HIV-related neurological conditions, ${ }^{16,17}$ and the impact of this reservoir on $\mathrm{TB}$ immunity in ART-treated subjects is unknown. ${ }^{18}$ Defects in myeloid dendritic cell and monocyte function may result in impaired cytokine production, which could render individuals more susceptible to TB.

The interleukin 1 (IL-1) and type 1 interferon (type 1 IFN) signaling pathways are well studied in mouse models but poorly understood in humans and have been shown to play opposing roles in Mtb host resistance. Type-1 IFNs contribute to pathogenesis through impairment of host resistance to $\mathrm{Mtb},{ }^{19,20}$ whereas $\mathrm{IL}-1 \beta$ is required for host control of infection. ${ }^{21}$ Thus, the balance between the IL-1 $\beta$ and type 1-IFN responses is pivotal for host survival during Mtb infection. In line with this work, recent reports in mouse models revealed that a mechanism behind the role of IL-1 in TB containment is mediated by the induction of prostaglandin E2 by IL-1, which limits production of type-1 IFNs. ${ }^{22}$ The study by Mayer-Barber and others provided proof of concept that therapies directed against the host innate inflammatory response are possible and can alter TB outcome. Given the fact that immunological features of TB differ in mice and humans (eg, characteristic tissue destruction preceding pulmonary cavitation is found in humans and not mice), validating these findings among HIV-infected patients most vulnerable to TB disease is critical.

We evaluated whether differences in innate immunological factors mediated protection or risk of TB recurrence through investigation of antigen presenting cell (APC) responses following stimulation with TLR ligands in Mtb/ HIV coinfected participants on ART with a previously defined history of successfully treated pulmonary TB.

\section{METHODS}

\section{Study Design and Cohort}

We conducted a nested case-control study among 24 virally suppressed HIV-infected patients from a larger prospective cohort of 520 subjects conducted between 2009 and 2014 investigating the incidence of TB recurrence following successful TB treatment among stable patients on ART, the TB Recurrence upon Successful Treatment for Tuberculosis and HIV (TRuTH) study. All participants previously enrolled in a CAPRISA trial investigating timing of ART initiation during treatment for pulmonary $\mathrm{TB}^{23}$ and with proven successful treatment for the previous TB episodes were eligible for enrolment into the TRuTH study. The TRuTH study screened participants quarterly over 60 months for TB recurrence, defined as first microbiologic confirmation of M. tuberculosis by TB smear or TB culture. A total of 12 cases and 12 controls were selected from the overall cohort. Matching criteria for cases and controls included age (within a 5-year window), gender, study arm assignment in the previous trial, previous history of TB, and baseline pre-ART CD4 count (within a 100 cells per microliter window). Written informed consent was obtained from all study participants before enrolment and the University of KwaZulu-Natal Biomedical Research Ethics Committee approved the study (ref: BF 051/09; Clin trials. gov number NCT 01539005). 
Peripheral blood mononuclear cell (PBMC) samples were collected prospectively and stored at 3-6 month intervals. Among the 12 cases, 2 time-points before TB recurrence were studied and compared with time-points from the matched control subjects. There were 42 sample visits for the 12 controls; whereas for the 12 cases, the number of sample visits in TB recurrent participants included pre-TB $(\mathrm{n}=16)$, untreated TB recurrence $(n=11)$, TB treatment $(n=13)$, and post-TB treatment $(n=4)$.

\section{In Vitro Stimulation of PBMCs With Toll-Like Receptor Ligands}

Cryopreserved PBMCs from cases and controls were thawed, resuspended at $1 \times 10^{6}$ cells per milliliter in R10 media [RPMI 1640 supplemented with $10 \%$ heat-inactivated fetal calf serum (FCS), $100 \mathrm{U} / \mathrm{mL}$ penicillin, $1.7 \mathrm{nM}$ sodium glutamate, and $5.5 \mathrm{~mL}$ HEPES buffer] and rested for 2 hours at $37^{\circ} \mathrm{C}, 5 \%$ $\mathrm{CO}_{2}$. One million cells were stimulated with pretitrated amounts of the following antigens: $1 \mu \mathrm{g} / \mathrm{mL}$ of heat-killed Listeria monocytogenes (TLR-2 HKLM), $1 \mu \mathrm{g} / \mathrm{mL}$ Lipoarabinomannans from Mycobacterium smegmatis (TLR-2, LAM-MS), $0.1 \mu \mathrm{g} / \mathrm{mL}$ of lipopolysaccharide (TLR-4, LPS-EK), $1 \mu \mathrm{g} / \mathrm{mL}$ thiazoloquinolone derivative (TLR-7/8, CL075), $75 \mathrm{ng} / \mathrm{mL}$ of Trehalose6,6-dibehenate (C-Type Lectin Receptor TDB), and $3 \mu \mathrm{g} / \mathrm{mL}$ of Bacillus Calmette-Guérin (BCG). All ligands were from Invivogen aside from BCG (Statens Serum Institute, Copenhagen, Denmark). Samples with viability above $50 \%$ were used in experimental assays. The viability was not statistically different in cases and controls median viability at baseline was $80 \%$ in cases and $64 \%$ in controls, $P=0.17$; and the median was $74 \%$ and $73 \%$ in cases and controls, respectively, for all time-points assessed $(P=0.43)$. A negative control tube with PBMCs in media alone was included in all assays, and for all analyses, cytokine production in stimulated samples was subtracted from the negative control with media alone. Brefeldin A $(5 \mu \mathrm{g} / \mathrm{mL}$; Sigma-Aldrich, St. Louis, MO) was added to all tubes immediately after adding the TLR ligands and stimulation of PBMCs was carried out for 18 hours at $37^{\circ} \mathrm{C}$ and $5 \% \mathrm{CO}_{2}$. Because of sample limitations, stimulation with TB-specific antigens LAM and TDB was performed in half of the participants.

\section{Flow Cytometry}

Following stimulation, PBMCs were washed with phosphate buffer saline (PBS) and stained for intracellular amine groups to differentiate live and dead cells using the aqua viability dye (Invitrogen, Camarillo, CA) for 20 minutes at $4^{\circ}$ C. Cells were then stained for 20 minutes at room temperature (RT) using monoclonal antibodies specific for the following surface markers: HLA-DR Pacific Blue (clone L243; Biolegend, San Diego, CA), CD14-APC CY7 (clone Mphip9), CD19Alexa Flour 700 (clone HIB19), CD56-Alexa Flour 700 (clone B159), CD3-Alexa Flour 700 (clone UCHT1), and CD11c-PE Cy5 (clone B-ly6) (all from BD Biosciences, San Diego, CA). Cells were then washed, fixed with Fix/Perm Medium A (Caltag, Frederick, MD), and incubated for 20 minutes at RT. Cells were washed again, permeabilized (Fix/Perm B; Caltag), and stained for intracellular expression of: $\mathrm{TNF} \alpha-$
PE Cy7 (clone MAb11), IL-12 (clone C11.5) APC, IL1 $\beta$ FITC (clone AS10), and IP10-PE (clone 6D4/D6/G2) (all from BD Biosciences) for 30 minutes at RT. Cells were finally washed and resuspended in PBS before acquisition on an LRSII flow cytometer. At least 500,000 events were acquired per sample and analyzed using the FlowJo software (version 9.4.11; TreeStar, Ashland, OR).

\section{Statistical Analyses}

To assess the predictive value of cytokine expression on TB recurrence, a generalized estimating equations (GEE) model was fitted to case-control status, using a binomial distribution, accounting for possible repeated measures and controlling for matched variables. Cytokine concentrations measured at pre-TB time-points were compared with all other time-points from those individuals who never experienced a TB recurrence. Longitudinal assessment of IL-1 $\beta$ changes in APCs in participants with TB recurrence was done using Wilcoxon matched-pairs signed ranked test. Differences between groups and cytokine expression were considered statistically significant at the $P<0.05$ level. Statistical analysis was performed using SAS version 9.3 (SAS Institute Inc., Cary, NC) and graphs were plotted using GraphPad Prism (version 5).

\section{RESULTS}

\section{Clinical Characteristics of Participant Groups}

The incidence rate of TB recurrence in the overall cohort was $4.07 \%$ (95\% CI: 3.24 to 5.06). The median CD4 count for the studied participants was not statistically different at 289 cells per cubic millimeter (IQR 105-470) for the cases and a median of 423 cells per cubic millimeter (IQR 335-536) for the controls $(P=0.2)$. The mean age was 34 years for the cases and 35 years for the controls and the cohort was predominantly female (67\%) irrespective of the arm. The majority of the subjects were clinically stable, had their viral loads suppressed, and had received ART for a median of 31 months (Table 1).

\section{APC Frequencies Between Cases and Controls}

We first assessed the frequencies of monocytes and myeloid dendritic cells (mDCs) in cases and controls. Monocytes were defined as $\mathrm{HLA}-\mathrm{DR}{ }^{+} \mathrm{CD} 11 \mathrm{c}^{+} \mathrm{CD} 14^{+}$and $\mathrm{mDCs}$ as $\mathrm{HLA}-\mathrm{DR}^{+} \mathrm{CD} 14^{-} \mathrm{CD} 11 \mathrm{c}^{+}$following gating on live cells and exclusion of $\mathrm{T}$ cells, $\mathrm{B}$ cells, and NK cells $\left(\mathrm{CD} 3^{-} \mathrm{CD} 19^{-} \mathrm{CD}^{-} 6^{-}\right)$, respectively (see gating strategy in Fig. 1A). The median baseline frequency of monocytes was $2.5 \%$ (IQR 2.1-7.6) in cases and $1.88 \%$ (IQR $0.4-4.1$ ) of HLA-DR-positive live dump-negative PBMCs in controls. We also evaluated the frequencies of monocytes following stimulation and neither of the comparisons were statistically significant, suggesting that the frequency of the monocytes was not predictors of TB outcome in this study $(P=0.14)$ without stimulation, or BCG $(P=0.2)$, LPS $(P=0.4)$, HKLM $(P=0.15)$, CL075 $(P=0.47)$ stimulated (data not shown).

In contrast, the median frequency of $\mathrm{mDCs}$ in cases was significantly higher than in controls at baseline and 
TABLE 1. Baseline Characteristics for Nested Case-Control Study Participants, Overall and Stratified by TB Recurrence Outcome

\begin{tabular}{|c|c|c|c|}
\hline Characteristic & All $(n=24)$ & TB Recurrence $(n=12)$ & No TB Recurrence $(n=12)$ \\
\hline Age at pre-ART, yrs, mean (SD); min-max & 34.7 (6.11); 24-47 & $34.2(5.73) ; 24-43$ & 35.2 (6.67); 24-47 \\
\hline Female, n (\%) & $16(66.7)$ & $8(66.7)$ & $8(66.7)$ \\
\hline Integrated arm, n (\%) & $16(66.7)$ & $8(66.7)$ & $8(66.7)$ \\
\hline Pre-ART CD4 count, cells $/ \mu \mathrm{L}$, mean (SD); min-max & $113(72.06) ; 11-237$ & $114(73.85) ; 11-237$ & $113(73.50) ; 11-230$ \\
\hline $\begin{array}{l}\text { Pre-ART log viral load, copies/mL, mean (SD); } \\
\text { min-max }\end{array}$ & $5.10(0.83) ; 2.60-6.18$ & $5.14(0.70) ; 3.62-6.15$ & $5.08(1.00) ; 2.60-6.18$ \\
\hline Current CD4 count, cells $/ \mu \mathrm{L}$, mean (SD); min-max & 453 (435.35); 14-2188 & 466 (604.73); 14-2188 & 439 (119.55); 271-671 \\
\hline Current CD4 count, cells $/ \mu \mathrm{L}$, median (IQR); $\min -\max$ & 354 (271-519); 14-2188 & $289(105-470) ; 14-2188$ & $423(335-536) ; 271-671$ \\
\hline Current viral load suppressed, $\dagger \mathrm{n} / \mathrm{N}(\%)$ & $19 / 23(82.6)$ & $8 / 12(66.7)$ & $11 / 11(100)$ \\
\hline Weight, kg, mean (SD) & $58.2(8.75)$ & $59.1(9.16)$ & $57.3(8.62)$ \\
\hline Body mass index, mean (SD) & $21.6(3.42)$ & $21.9(3.62)$ & $21.4(3.36)$ \\
\hline \multicolumn{4}{|l|}{ Cavitory disease at occurrence, $\mathrm{n}(\%)$} \\
\hline No & $0(0.0)$ & $0(0.0)$ & $0(0.0)$ \\
\hline One lung & $7(29.2)$ & $1(8.3)$ & $6(50.0)$ \\
\hline Both lungs & $17(70.8)$ & $11(91.7)$ & $6(50.0)$ \\
\hline \multicolumn{4}{|l|}{ Adenopathy at occurrence, $\mathrm{n}(\%)$} \\
\hline No & $23(95.8)$ & $12(100)$ & $11(91.7)$ \\
\hline One lung & $1(4.2)$ & $0(0.0)$ & $1(8.3)$ \\
\hline Both lungs & $0(0.0)$ & $0(0.0)$ & $0(0.0)$ \\
\hline \multicolumn{4}{|l|}{ Pleural infusion at occurrence, $\mathrm{n}(\%)$} \\
\hline No & $21(87.5)$ & $12(100)$ & $9(75.0)$ \\
\hline One lung & $3(12.5)$ & $0(0.0)$ & $3(25.0)$ \\
\hline Both lungs & $0(0.0)$ & $0(0.0)$ & $0(0.0)$ \\
\hline
\end{tabular}

*One TB recurrence case was not on ARVs at the time of enrolment on TRUTH and the pre-TB time-point was a month before initiating treatment.

†One patient, who did not experience TB recurrence, did not have VL data available at the first time-point measured. Current refers to the very first time-point; CD4 and VL were measured and duration of time on ARVs in this substudy.

following antigen stimulation, suggesting that this could be an indicator to predict TB recurrence. At baseline, $\mathrm{mDCs}$ were detected at $61.1 \%$ (IQR 50.3-68.6) and 33.8\% (IQR 20.7-57.3) of HLA-DR-positive live dump-negative PBMCs in controls $(P=0.04$ for unstimulated, $P=0.02$ for BCG, $P=0.02$ for LPS, $P=0.008$ for HKLM and $P=$ 0.01 for CL075 stimulation, data not shown).

\section{Decreased IL-1 $\beta$ and TNF- $\alpha$ Expression in Monocytes is Associated With the Risk of TB Recurrence}

We next assessed whether APC function, particularly early cytokine production signatures in both monocytes and $\mathrm{mDCs}$ of cases and controls, would predict $\mathrm{TB}$ recurrence. We initially analyzed the predictive time-point (closest to TB recurrence) in cases compared with the matched time-point in the controls and found lower cytokine production in cases than in controls; however, these differences were not statistically significant (data not shown). Next, a generalized estimating equations (GEE) model using binomial distribution was fitted to case-control status and corrected for repeated measures and matched variables. Only cases sampled at 2 time-points before TB recurrence and all control samples were analyzed. We found that increased IL-1 $\beta$ expression was significantly associated with protection from TB recurrence when stimulated with several antigens including LPS, HKLM, CL075 and that this response was mostly mediated by monocytes (Table 2 and Fig. 1B). For every $1 \%$ increase in IL-1 $\beta$ (in response to LPS stimulation) in monocytes, the odds of TB recurrence decreased by $6 \%$ (OR 0.94; 95\% CI: 0.89 to $1.00, P=0.04$, Table 2). Similar data were observed for HKLM (OR 0.96; 95\% CI: 0.92 to $1.00, P=0.05$ ) and CL075 (OR 0.95; 95\% CI: 0.91 to 0.99 , $P=0.02)$. The expression of TNF- $\alpha$, another proinflammatory innate mediator, was also decreased in monocytes following LPS stimulation in individuals who experienced TB recurrence $(P=0.03$, Table 2$)$.

Interestingly, no similar observations were noted with regard to IL- $1 \beta$ production in $\mathrm{mDCs}$ following stimulation with multiple TLR stimulations with the exception of TDB (Supplemental Digital Content, Table 1, http://links.lww. com/QAI/A926), suggesting that this IL-1 $\beta$ defect in the cases is mediated by the monocytes. We instead observed 
FIGURE 1. Representative gating strategy and measurement of all cytokines (IL-1 $\beta$, IP10, TNF- $\alpha$, and IL-12) in monocytes (HLA-DR ${ }^{+} \mathrm{CD} 11 \mathrm{c}^{+} \mathrm{CD} 14^{+}$) and myeloid dendritic cells (mDCs;

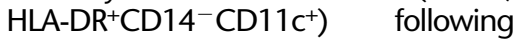
stimulation with TLR-7/8 ligand (CL075). A, Gating was initially on lymphocytes followed by exclusion of cell doublets; dead cells, T cells, B cells, and NK cell exclusion using the live/ dead exclusion dye and CD3, CD19, and CD56 markers, respectively, followed by gating on HLA-DR-positive cells. PBMCs were either unstimulated or stimulated with TLR-7/8 ligand (CL075) and all cytokine responses are shown for monocytes (HLA$\left.\mathrm{DR}^{+} \mathrm{CD} 11 \mathrm{c}^{+} \mathrm{CD} 14^{+}\right)$, for first 2 rows, and (mDCs; HLA-DR+CD14-CD11 ${ }^{+}$) for the last 2 rows in a control subject without TB recurrence (no TB) shown on the left panel or in an individual who experienced TB recurrence (case) shown of the right panel. Monocytes and $\mathrm{mDC}$ gates were each derived from the lineage negative followed by gating on HLA-DR-positive cells. Initial representative gating strategy (top row) refers to an unstimulated sample. B, Representative predictive (0-6 months and 6 months time-points) cytokine (IL$1 \beta$ ) response profiles in monocytes (top panel) and mDCs (bottom panel) of individuals with TB recurrence (cases) during successful treatment of TB before TB recurrence (TB) versus controls (NTB) over time. Only cases sampled before TB recurrence ( $0-6$ months and 6 months time-points) were analyzed and control samples. Box and whiskers represent individual IL-1 $\beta$ responses in cases (red) and controls (blue, for monocytes or green for $\mathrm{mDCs})$; the $P$-value refers to differences in IL-1 $\beta$ production between cases and controls at the predictive time-points [also referred to in Table 2 (monocytes) and Supplemental Digital Content, Table 1, http://links.lww.com/QAl/ A926 (mDCs)] (C and D). Longitudinal assessment of IL-1 $\beta$ changes in monocytes (C) and mDCs (D) at pre-TB $(n=16)$, during $(n=11)$, and post-TB $(n=17)$ time-points in the TB recurrence group. Assessment of $\mathrm{IL}-1 \beta$ changes in monocytes and mDCs of participants with TB recurrence was done using Wilcoxon matched-pairs signed ranked test. Lines represent median with interquartile range. Fewer time-points were analyzed for monocytes compared with mDCs because of cell availability from cryopreserved PBMC.
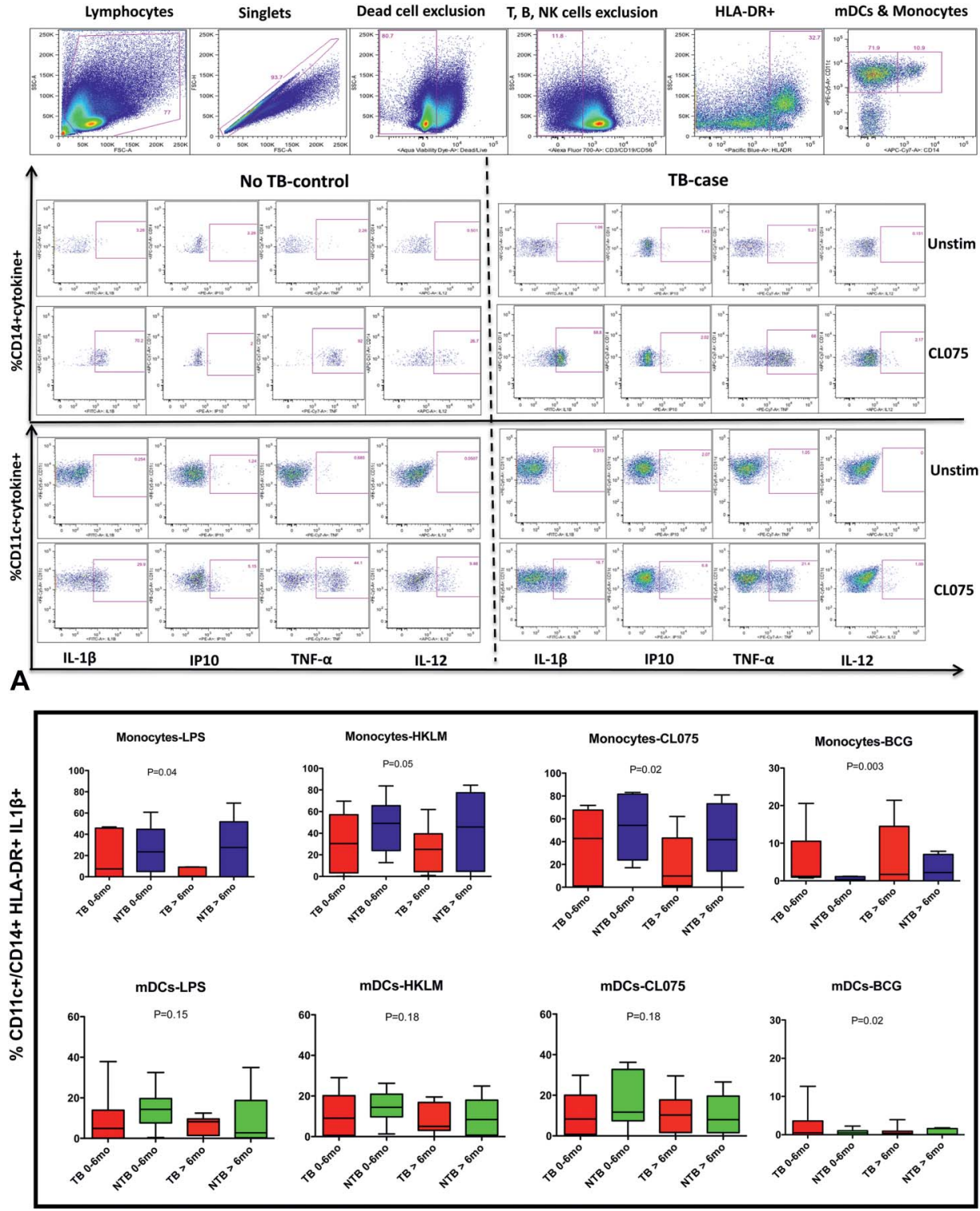

B

Time to Recurrence/Endpoint
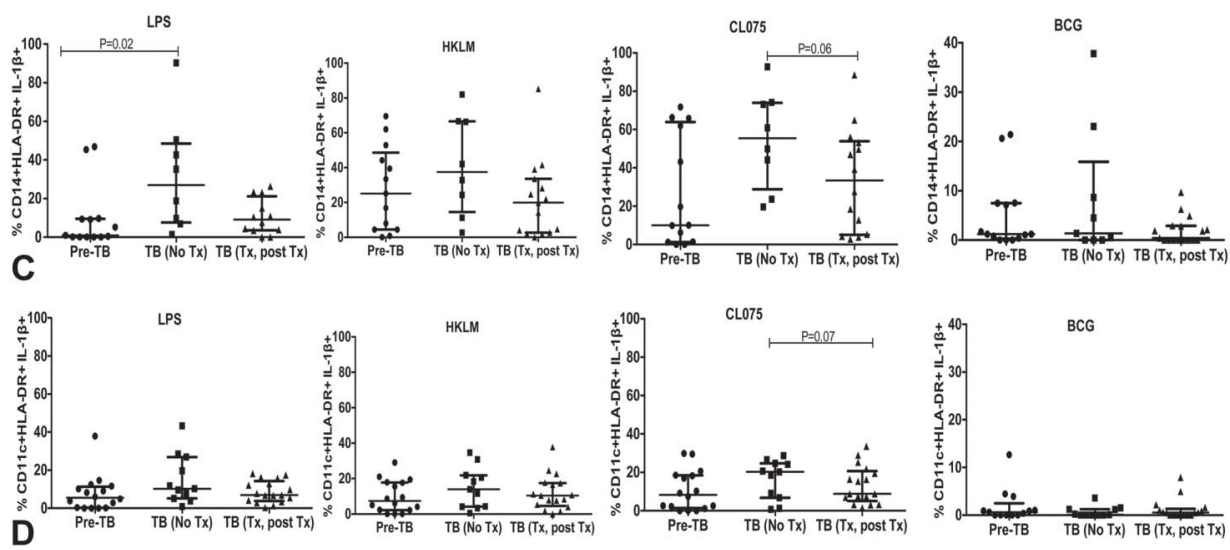
TABLE 2. Association of Monocyte Cytokine Responses With Susceptibility to TB Recurrence

\begin{tabular}{|c|c|c|c|c|c|c|}
\hline \multirow[b]{2}{*}{ Cytokine } & \multicolumn{2}{|c|}{ No TB Recurrence } & \multicolumn{2}{|c|}{ TB Recurrence } & \multirow[b]{2}{*}{ Unadjusted Odds Ratio (95\% CI) } & \multirow[b]{2}{*}{$\boldsymbol{P}$} \\
\hline & $\overline{\mathbf{N}}$ & Median (IQR) & $\overline{\mathbf{N}}$ & Median (IQR) & & \\
\hline \multicolumn{7}{|c|}{ Monocytes (CD14) } \\
\hline \multicolumn{7}{|c|}{ Antigen: BCG } \\
\hline IL-12 & 36 & $0.01(0.00-0.51)$ & 9 & $0.08(0.00-0.34)$ & $0.40(0.01$ to 11.15$)$ & 0.5901 \\
\hline IP10 & 36 & $0.13(0.00-1.14)$ & 9 & $0.00(0.00-1.34)$ & $1.53(0.84$ to 2.77$)$ & 0.1640 \\
\hline $\mathrm{TNF}-\alpha$ & 36 & $1.19(0.00-3.78)$ & 9 & $1.43(0.38-5.41)$ & $1.06(0.78$ to 1.44$)$ & 0.7162 \\
\hline \multicolumn{7}{|c|}{ Antigen: LPS } \\
\hline IL-1 $\beta$ & 34 & $21.03(0.00-45.51)$ & 11 & $0.92(0.00-9.28)$ & $0.94(0.89$ to 1.00$)$ & 0.0462 \\
\hline IL-12 & 33 & $1.85(0.00-7.28)$ & 9 & $3.08(1.31-7.69)$ & $0.98(0.85$ to 1.13$)$ & 0.8143 \\
\hline IP10 & 33 & $0.00(0.00-1.15)$ & 9 & $0.18(0.00-2.86)$ & $0.94(0.84$ to 1.05$)$ & 0.2807 \\
\hline TNF- $\alpha$ & 33 & $57.44(23.10-73.02)$ & 9 & $41.80(20.95-61.51)$ & $0.95(0.91$ to 1.00$)$ & 0.0316 \\
\hline \multicolumn{7}{|c|}{ Antigen: HKLM } \\
\hline IL-1 $\beta$ & 38 & $45.45(23.93-70.23)$ & 11 & $25.11(4.44-52.85)$ & $0.96(0.92$ to 1.00$)$ & 0.0564 \\
\hline \multicolumn{7}{|c|}{ Antigen: CL075 } \\
\hline IL-1 $\beta$ & 36 & $51.16(32.04-73.55)$ & 11 & $10.05(1.25-62.00)$ & $0.95(0.91$ to 0.99$)$ & 0.0243 \\
\hline IL-12 & 35 & $6.42(2.02-15.30)$ & 9 & $11.29(9.76-18.65)$ & $1.02(0.96$ to 1.08$)$ & 0.5811 \\
\hline IP10 & 35 & $0.09(0.00-1.88)$ & 9 & $0.54(0.00-1.54)$ & $1.61(0.80$ to 3.24$)$ & 0.1815 \\
\hline TNF- $\alpha$ & 35 & $62.88(46.20-76.72)$ & 9 & $62.71(24.45-77.10)$ & $0.99(0.94$ to 1.03$)$ & 0.4948 \\
\hline \multicolumn{7}{|c|}{ Antigen: LAMMS } \\
\hline IL-1 $\beta$ & 16 & $34.81(28.60-56.89)$ & 5 & $12.50(0.00-17.21)$ & N/A & \\
\hline IL-12 & 15 & $1.19(0.33-1.42)$ & 2 & $1.40(0.95-1.85)$ & N/A & \\
\hline IP10 & 15 & $0.00(0.00-0.75)$ & 2 & $0.08(0.00-0.16)$ & N/A & \\
\hline TNF- $\alpha$ & 15 & $40.83(28.90-52.18)$ & 2 & $43.49(34.22-52.75)$ & N/A & \\
\hline \multicolumn{7}{|c|}{ Antigen: TDB } \\
\hline IL-1 $\beta$ & 12 & $19.05(8.97-33.42)$ & 5 & $0.00(0.00-6.33)$ & N/A & \\
\hline
\end{tabular}

Cytokine expression in monocytes from the cases before TB acquisition and controls over time was analyzed using the generalized estimating equations (GEE) model correcting for repeated measures and matched variables. Bold values indicates significant differences in cytokine expression between TB recurrence and no recurrence groups.

that BCG stimulation was associated with $42 \%$ and $30 \%$ increased risk of TB recurrence per $1 \%$ increase in IL-1 $\beta$ expression in both mDCs and monocytes (OR $1.42 ; 95 \% \mathrm{CI}$ : 1.04 to $1.95 ; P=0.02$; OR $1.30 ; 95 \% \mathrm{CI}: 1.09$ to $1.55 ; P=$ 0.003 ; in mDCs and monocytes, respectively, Supplemental Digital Content, Table 1, http://links.lww.com/QAI/A926 and Table 2). In addition, we also noted a similar pattern of increased odds of TB recurrence with IL-12 production in response to HKLM stimulation only in $\mathrm{mDCs}$, but not in response to other antigens or in monocytes. We did not observe any association of IP10 with TB recurrence.

\section{Longitudinal Changes in IL-1 $\beta$ Expression Amongst Subjects With Recurrent TB}

We next investigated longitudinal changes in IL-1 $\beta$ levels beyond the predictive time-points in the TB recurrent group. Our findings showed a significant increase in IL-1 $\beta$ production on monocytes following LPS stimulation and only nonsignificant increase was noted for other stimulations during TB; however, these levels decreased following TB treatment suggesting that antigen load mediated the observed increase in the studied individuals. Differences were noted pre-TB and during TB recurrence following stimulation with LPS $(P=0.02)$ and a trend toward decreased IL-1 $\beta$ production was observed following CL075 stimulation during TB and post-TB treatment $(P=0.06)$. In addition, a trend toward higher IL-1 $\beta$ production was noted for CL075 stimulation following onset of $\mathrm{TB}$ recurrence and this decreased post-TB treatment $(P=0.07)$ in mDCs (Figs. 1C, D).

Taken together, our data show that the observed elevated production of IL-1 $\beta$ from monocytes following several antigen stimulations with TLR-2, TLR-4, and TLR $7 / 8$ may be associated with odds ratios of protection from TB recurrence and may therefore suggest that the status of the innate immune system, particularly impaired APC responses to TLR stimulation, may predict susceptibility to TB disease. 


\section{DISCUSSION}

Factors that mediate protective immunity against Mtb are not fully understood and involve both innate and adaptive mechanisms. There is a need to identify biomarkers that accurately predict the risk of $\mathrm{TB}$ recurrence following successful treatment of TB and define underlying immune mechanisms that may serve as vaccine or therapeutic targets. This study aimed to address this gap by investigating innate immune factors prospectively linked to TB outcomes. In particular, we investigated whether the functional status of antigen presenting cells confers protection or risk from TB recurrence among stable $\mathrm{HIV}$-infected patients accessing ART with previous history of successful TB therapy.

Our findings show that of all cytokines tested following TLR stimulation; the overall expression of IL- $1 \beta$, and to a lesser extent TNF- $\alpha$, from monocytes was the best predictor of TB recurrence. We show that the cytokine production defect as demonstrated by lower IL-1 $\beta$ frequencies in the cases than in the controls was consistent across multiple TLR stimulations and may indicate a functional defect in monocytes following stimulation with TLR ligands. In addition, we demonstrated that APC responses to BCG stimulation were associated with increased risk of TB recurrence and may mark an important difference in innate host response to TB in cases compared with controls.

Phagocytes, such as macrophages and dendritic cells, are the first line of defense against TB, engulfing the bacilli and (if successful) limiting the severity of infection. They also alert the host to the presence of infection through pattern recognition of $\mathrm{Mtb}$, thus coordinating the innate and subsequent adaptive host immune responses. The importance of IL-1 $\beta$ in mediating host response against TB has been previously reported, ${ }^{24}$ and recent reports show that IL-1 $\beta$ directly augments TNF-signaling, up-regulates TNF secretion and TNFR1 cell surface expression leading to caspase-3 activation, apoptosis, and direct killing of Mtb in macrophages. $^{25}$ Data from animal models and human clinical studies describe a network of pathways involving immune regulatory molecules that increase the risk of developing TB among which interleukins and interferons feature prominently. ${ }^{20,21,24,26,27}$ Our findings on the IL-1 $\beta$ differences at the onset of $\mathrm{TB}$ are consistent with a report from a recent study conducted in mice infected with TB. The study demonstrated the importance of IL-1 in reducing disease severity through the induction of prostaglandin E2 levels (PGE2) that limit the production of type-I interferons, which are associated with increased TB disease severity. ${ }^{22}$ The authors provided proof of concept for host-directed TB therapy and a potential for alternative options for TBinfected individuals in the absence of a vaccine. We here make similar observations on the possible role of IL-1 $\beta$ in mediating TB containment; however, the mechanism behind this defect or augmentation with some antigen stimulations requires further investigation in our cohort and in untreated HIV-infected and HIV-uninfected cohorts.

There are several other factors that could affect the innate immune system's decreased capacity to respond, precipitating TB recurrence. Firstly, systemic inflammation that is not fully suppressed despite successful ART may be an indication of low-level HIV-1 viremia and microbial translocation, which could be sources of continuous in vivo TLR stimulation of APCs. Secondly, Mtb can escape host recognition by inducing type 1 IFN that inhibits IL-1 $\beta$ release by macrophages and dendritic cells through suppression of IL-1 $\beta$ at transcriptional mRNA level. ${ }^{28,29}$ Expansion of patrolling monocytes with unusual phenotypes (CD16 expression and low levels of CD14) has been reported to be induced by HIV infection even in the presence of ART resulting in continuous TLR stimulation by the virus. ${ }^{30}$ Host genetic factors may play a role in influencing the nature of the generated immune response ${ }^{31,32}$ accounting for the differences in outcome between the 2 study groups. Lastly, a defect in innate immunity as suggested by our findings or induced by any of the listed possible mechanisms could result in failure to induce $\mathrm{CD}^{+}$T-cell-mediated immune responses well known to be fundamental to the control of Mtb. The above listed are some of the possible mechanisms that warrant follow-up investigation using this cohort. It was surprising to note that $\mathrm{mDCs}$ did not demonstrate a clear predictive outcome of TB risk with several antigens as noted for monocytes. Studies show that TB could induce impairment of dendritic cell maturation and increase IL-10 production known to suppress T-cell response resulting in an imbalance of IFN- $\gamma$, enhanced IL6 and IL-10 production, and other causes implicated in low antigen-specific response and function associated with TB infection. ${ }^{33-35}$ It is possible that the response of $\mathrm{mDCs}$ and monocytes to BCG as a risk factor for $\mathrm{TB}$ could be a preclinical response. Extremes in the ratio of peripheral blood to monocyte lymphocytes were found to be associated with increased risk of TB in HIV-infected adults in South Africa and suggested to be a tool to stratify risk of TB. ${ }^{36}$ In a separate study in infants who received $B C G$ vaccination at birth and had higher monocyte to T-cell ratios, transcriptional profiles were associated with an activated macrophage phenotype likely involved in pathogenesis of risk of TB disease suggesting that a phenotype of activated monocytes may be detrimental to the host. ${ }^{37}$ It is thus important to classify the monocyte subsets behind this IL-1 $\beta$ response in further studies and measure associated soluble markers of inflammation.

The ability of monocyte/macrophages and myeloid dendritic cells (mDCs) to modulate immune responses relevant to TB immunity has been demonstrated. ${ }^{38,39}$ The long TLR stimulations employed in our study, the low frequency, and identification of monocytes from frozen samples are some of the study limitations that warrant further interrogation in future studies. Nevertheless, our findings from a well-described prospective cohort of HIV/TB coinfected individuals following multiple TLR stimulations of innate cells suggest that innate immune signaling may be an important predictor of TB pathogenesis. In particular, production of IL-1 $\beta$ by innate immune cells following TLR and BCG stimulations correlated with differential TB recurrence outcomes in ART-treated patients and highlights differences in host response to TB. Although this is a pilot study with a very small participant sample size and needs to be validated 
in a larger cohort, with accompanying exploration of the innate mechanisms, it nonetheless supports recent findings and provides evidence in humans that IL-1 $\beta$ levels may have an impact on reactivation of TB. The study may therefore have public health implications calling for a need to identify individuals at risk for $\mathrm{TB}$ reactivation for host-directed therapies to reverse or reduce $\mathrm{TB}$ severity in $\mathrm{TB}$ endemic areas in the future.

\section{ACKNOWLEDGMENTS}

The authors would like to thank the CAPRISA clinic team and participants of the SAPIT and TRUTH study at the eThekwini Clinic.

\section{REFERENCES}

1. Chaisson RE, Martinson NA. Tuberculosis in Africa-combating an HIV-driven crisis. $N$ Engl J Med. 2008;358:1089-1092.

2. Corbett EL, Watt CJ, Walker $\mathrm{N}$, et al. The growing burden of tuberculosis: global trends and interactions with the HIV epidemic. Arch Intern Med. 2003;163:1009-1021.

3. Glynn JR, Murray J, Bester A, et al. High rates of recurrence in HIVinfected and HIV-uninfected patients with tuberculosis. J Infect Dis. 2010;201:704-711

4. Sonnenberg P, Murray J, Glynn JR, et al. HIV-1 and recurrence, relapse, and reinfection of tuberculosis after cure: a cohort study in South African mineworkers. Lancet. 2001;358:1687-1693.

5. Verver S, Warren RM, Beyers N, et al. Rate of reinfection tuberculosis after successful treatment is higher than rate of new tuberculosis. $\mathrm{Am} \mathrm{J}$ Respir Crit Care Med. 2005;171:1430-1435.

6. Cosma CL, Sherman DR, Ramakrishnan L. The secret lives of the pathogenic mycobacteria. Annu Rev Microbiol. 2003;57:641-676.

7. Diedrich CR, Flynn JL. HIV-1/mycobacterium tuberculosis coinfection immunology: how does HIV-1 exacerbate tuberculosis? Infect Immun. 2011;79:1407-1417.

8. Kaufmann SH. Tuberculosis vaccines: time to think about the next generation. Semin Immunol. 2013;25:172-181.

9. Monack DM, Mueller A, Falkow S. Persistent bacterial infections: the interface of the pathogen and the host immune system. Nat Rev Microbiol. 2004;2:747-765.

10. Aaron L, Saadoun D, Calatroni I, et al. Tuberculosis in HIV-infected patients: a comprehensive review. Clin Microbiol Infect. 2004;10: 388-398.

11. Hossain MM, Norazmi MN. Pattern recognition receptors and cytokines in Mycobacterium tuberculosis infection - the double-edged sword? Biomed Res Int. 2013;2013:179174.

12. Akamine M, Higa F, Arakaki N, et al. Differential roles of Toll-like receptors 2 and 4 in in vitro responses of macrophages to Legionella pneumophila. Infect Immun. 2005;73:352-361.

13. Poltorak A, He X, Smirnova I, et al. Defective LPS signaling in $\mathrm{C} 3 \mathrm{H} / \mathrm{HeJ}$ and C57BL/10ScCr mice: mutations in Tlr4 gene. Science. 1998;282: 2085-2088.

14. Horne DJ, Randhawa AK, Chau TT, et al. Common polymorphisms in the PKP3-SIGIRR-TMEM16J gene region are associated with susceptibility to tuberculosis. J Infect Dis. 2012;205:586-594.

15. Lawn SD, Myer L, Edwards D, et al. Short-term and long-term risk of tuberculosis associated with CD4 cell recovery during antiretroviral therapy in South Africa. AIDS. 2009;23:1717-1725.

16. Cobos-Jimenez V, Booiman T, Hamann J, et al. Macrophages and HIV-1. Curr Opin HIV AIDS. 2011;6:385-390.

17. Gavegnano C, Schinazi RF. Antiretroviral therapy in macrophages: implication for HIV eradication. Antivir Chem Chemother. 2009;20: 63-78.
18. Walker NF, Meintjes G, Wilkinson RJ. HIV-1 and the immune response to TB. Future Virol. 2013;8:57-80.

19. Stanley SA, Johndrow JE, Manzanillo P, et al. The Type I IFN response to infection with Mycobacterium tuberculosis requires ESX1-mediated secretion and contributes to pathogenesis. J Immunol. 2007; 178:3143-3152.

20. Manca C, Tsenova L, Bergtold A, et al. Virulence of a Mycobacterium tuberculosis clinical isolate in mice is determined by failure to induce Th1 type immunity and is associated with induction of IFN-alpha/beta. Proc Natl Acad Sci U S A. 2001;98:5752-5757.

21. Fremond CM, Togbe D, Doz E, et al. IL-1 receptor-mediated signal is an essential component of MyD88-dependent innate response to Mycobacterium tuberculosis infection. J Immunol. 2007;179:1178-1189.

22. Mayer-Barber KD, Andrade BB, Oland SD, et al. Host-directed therapy of tuberculosis based on interleukin-1 and type I interferon crosstalk. Nature. 2014;511:99-103.

23. Abdool Karim SS, Naidoo K, Grobler A, et al. Integration of antiretroviral therapy with tuberculosis treatment. $N$ Engl J Med. 2011; 365:1492-1501.

24. Mayer-Barber KD, Barber DL, Shenderov K, et al. Caspase-1 independent IL-1beta production is critical for host resistance to mycobacterium tuberculosis and does not require TLR signaling in vivo. J Immunol. 2010;184:3326-3330.

25. Jayaraman P, Sada-Ovalle I, Nishimura T, et al. IL-1beta promotes antimicrobial immunity in macrophages by regulating TNFR signaling and caspase-3 activation. J Immunol. 2013;190:4196-4204.

26. Berry MP, Graham CM, McNab FW, et al. An interferon-inducible neutrophil-driven blood transcriptional signature in human tuberculosis. Nature. 2010;466:973-977.

27. Manca C, Tsenova L, Freeman S, et al. Hypervirulent M. tuberculosis W/Beijing strains upregulate type I IFNs and increase expression of negative regulators of the Jak-Stat pathway. J Interferon Cytokine Res. 2005;25:694-701.

28. Novikov A, Cardone M, Thompson R, et al. Mycobacterium tuberculosis triggers host type I IFN signaling to regulate IL-1beta production in human macrophages. J Immunol. 2011;187:2540-2547.

29. Remoli ME, Giacomini E, Lutfalla G, et al. Selective expression of type I IFN genes in human dendritic cells infected with Mycobacterium tuberculosis. J Immunol. 2002;169:366-374.

30. Cros J, Cagnard N, Woollard K, et al. Human CD14dim monocytes patrol and sense nucleic acids and viruses via TLR7 and TLR8 receptors. Immunity. 2010;33:375-386

31. Bellamy R. Susceptibility to mycobacterial infections: the importance of host genetics. Genes Immun. 2003;4:4-11.

32. Moller M, de Wit E, Hoal EG. Past, present and future directions in human genetic susceptibility to tuberculosis. FEMS Immunol Med Microbiol. 2010;58:3-26.

33. Hanekom WA, Mendillo M, Manca C, et al. Mycobacterium tuberculosis inhibits maturation of human monocyte-derived dendritic cells in vitro. $J$ Infect Dis. 2003;188:257-266.

34. Sakhno LV, Shevela EY, Tikhonova MA, et al. Impairments of antigenpresenting cells in pulmonary tuberculosis. J Immunol Res. 2015;2015: 793292.

35. Wolf AJ, Linas B, Trevejo-Nunez GJ, et al. Mycobacterium tuberculosis infects dendritic cells with high frequency and impairs their function in vivo. J Immunol. 2007:179:2509-2519.

36. Naranbhai V, Hill AV, Abdool Karim SS, et al. Ratio of monocytes to lymphocytes in peripheral blood identifies adults at risk of incident tuberculosis among HIV-infected adults initiating antiretroviral therapy. $J$ Infect Dis. 2014;209:500-509.

37. Fletcher HA, Filali-Mouhim A, Nemes E, et al. Human newborn bacille Calmette-Guerin vaccination and risk of tuberculosis disease: a casecontrol study. BMC Med. 2016;14:76

38. Akira S, Hemmi H. Recognition of pathogen-associated molecular patterns by TLR family. Immunol Lett. 2003;85:85-95.

39. Takeda K, Akira S. Toll-like receptors in innate immunity. Int Immunol. $2005 ; 17: 1-14$ 